developing our immunization strategies, these risks and costs must be weighed against the potential benefits of immunization.

Hepatitis B vaccine truly is a "light at the end of the tunnel." The availability of this vaccine will have a profound effect on the health care of hospital employees, preventive practices of physicians and the natural history of hepatitis B and its complications. In the long run, it may have profound effects on reducing the morbidity and mortality of primary liver cell carcinoma in countries where this disease is endemic. We must keep our eyes and minds open; be aware of the cost implications of vaccination; and analyze and reanalyze new data regarding vaccine efficacy and complications as they become available. For each potential recipient of vaccine we must weigh the benefits and the risks of vaccination and consider alternative approaches such as deferred or postexposure immunization. The development of hepatitis B vaccine and field application is exciting. But, like other important breakthroughs in science or medicine, this new advance also will bring with it new problems.

Richard A. Garibaldi, M.D. Associate Professor of Medicine Division of Infectious Diseases The University of Connecticut Health Center Farmington, Connecticut

\section{Hepatitis B Vaccine- A Boon for Whom?}

A killed vaccine to prevent hepatitis $B$ virus (HBV) infection (Heptavax-B, Merck, Sharp and Dohme) has been licensed and hailed as a major triumph. ${ }^{1,2}$ The vaccine will soon be widely available. In its trials thus far the vaccine appears to be antigenic, safe, and effective in preventing $\mathrm{HBV}$ infection. It undoubtedly will play an important role in reducing the rate of $\mathrm{HBV}$ infections in very high risk groups within the community (homosexuals, family contacts of patients with acute $\mathrm{HBV}$ infection, etc.).

While this vaccine certainly represents a major technological achievement, limited experience with the use of the vaccine in people, its expense, and a paucity of hard data on the annual risk of $\mathrm{HBV}$ infection within groups of workers in acute care hospitals will dictate considerably narrower application in its initial use in hospitals. ${ }^{1} \mathrm{~A}$ brief discussion of the factors which most affect decisions on vaccination of hospital workers follows.

The most pressing question is which individuals within a hospital should be vaccinated. One answer to this hinges on the question of which individuals within a hospital are at higher risk of acquiring hepatitis $B$ infection than individuals of the same age, race, socioeconomic status and sexual preference who are not hospital employees. Data to answer this question are not available since no incidence studies which include appropriate community controls have been done in hospital workers.

\footnotetext{
Address reprint requests to: Bruce H. Hamory, M.D., Assistant Projessor, Department of Medicine, Hospital Epidemiologist, L'niversity of Missouri Health Science Center, Columbia, MO 65212.
}

Another solution is to pick those individuals within the hospital who are at highest risk of $\mathrm{HBV}$ infection relative to other hospital workers. Most information on HBV infection in hospitals is not of high quality. However, there are three papers which report annual incidence rates for $\mathrm{HBV}$ infection among hospital employees in non-outbreak situations. Hirschowitz et al $^{3}$ reported an apparent attack rate of $3 \%$ per year in 129 clinical laboratory personnel followed serially over a twoyear period. Craig et $\mathrm{al}^{4}$ found the same rate of seroconversion to $\mathrm{HBV}$ markers among phlebotomists (9/270 person-months) as among secretaries ( $3 / 94$ personmonths) for an annual rate of under $1 \%$ per year. However, they noted a decrease in the rate of seroconversion as the study progressed, indicating a possible influence of the study on the rate. The Centers for Disease Control has just issued incidence data from hemodialysis staff ${ }^{5}$ with an HBV seroconversion rate of $2.6 \%$ per year. All other published studies of HBV markers in hospital staff have either reported prevalence rates only, or have been part of hepatitis outbreak investigations.

Before recommending vaccination of specific groups of hospital workers, it is helpful to briefly review other nonmonetary considerations against vaccination of healthy individuals at low risk of exposure to infection. These considerations may well include most hospital staff.

The first issue to be addressed is that of any rare but severe side effects of vaccination. There is minimal data to address this question since only 4,000 people have received $\mathrm{HBV}$ vaccine so far, and these have been carefully screened and selected for lack of HBV markers. 'T wo kinds of effects might be expected. The first is the occurrence of unusual reactions seen with natural $\mathrm{HBV}$ infection such as transverse myelitis or polyarteritis nodosa. The second type of reaction could be more severe disease occurring when a vaccinated person is exposed to natural infection during the waning phases of immunity. ${ }^{6}$ Such reactions have occurred with inactivated RSV, mycoplasma and measles vaccines. ${ }^{7-9}$ Since the role of hypersensitivity in liver injury seen with $\mathrm{HBV}$ infection is still unclear, ${ }^{6}$ and relatively few vaccinees have been followed long enough to evaluate what will happen when they are exposed to natural $\mathrm{HBV}$ infection in the waning phases of immunity, this remains an active concern. It is certainly true that neither of these potential problems, nor other potential pitfalls of vaccine made from human blood (extraneous viruses, etc.) have been observed. However, the small groups of vaccinees followed are not large enough to exclude these uncommon events with any certainty, as was demonstrated during the swine flue vaccination program.

This vaccine is an excellent immunogen, producing high titers of anti- $\mathrm{Hb}_{\mathrm{s}}$ following three doses in $95 \%$ of normal individuals. ${ }^{10}$ Because of this high response rate, it is not currently anticipated that vaccinees should have antibody titers performed following vaccination. However, one wonders whether an individual who does not respond to $\mathrm{HBV}$ vaccination should continue to work in a "high-risk area" for HBV infection. Will national designation of certain job locations within a hospital as "high-risk" pose any additional legal or moral burdens on the institution and its infection control personnel? In addition, is $95 \%$ confidence enough to forestall either a 
booster dose, a check of antibody status, or administration of HBIG following documented parenteral exposure to $\mathrm{HBV}$ contaminated blood?

Some considerations are the extremely high cost of vaccination ( $\sim \$ 100$ per person), the need for more data on side effects collected from larger groups of vaccinees, and the suggestion that cost benefit analyses produce cost savings to society from $\mathrm{HBV}$ vaccination only when the annual incidence of $\mathrm{HBV}$ is $2 \%$ or more. With these in mind, it seems logical to restrict initial vaccination efforts within acute care hospitals to personnel employed in hemodialysis units, clinical chemistry, serology labs, and blood banks. In these areas, incidence rates of $3 \%$ per year have been shown. Hard incidence data have not been published for other groups, but vaccination of operating room personnel, practicing surgeons, anesthesiologists, and pathologists, emergency room staff and possibly phlebotomists, seems defensible. I do not think that current data support the use of $\mathrm{HBV}$ vaccine in nonsurgical physicians, in general ward medicine, surgery, pediatric nurses, or among housekeeping, $\mathrm{x}$-ray, laundry, inhalation therapy or other ancillary health personnel, who are based in acute care hospitals. The above suggestions are made in reference to data from Pattison ${ }^{11}$ and others suggesting that frequent exposure to blood is the major risk factor for $\mathrm{HBV}$ infection in hospital personnel.

The HBV vaccine will not appreciably diminish the risk of exposure to $\mathrm{HBV}$ among hospital personnel for many years, if ever, since fully $50 \%$ of reported cases of $\mathrm{HBV}$ infection in a community are sporadic and some high-risk groups may prove difficult to vaccinate. It may offer protection against clinical disease and the severe sequelae of infection of small groups of hospital workers who are at a higher than usual risk of exposure to, and acquisition of, HBV infection in the hospital setting. Vaccination will not relieve hospitals of the need to isolate patients with hepatitis, to identify chronic carriers, or to handle blood products carefully because immunity can be overwhelmed by massive inocula, and because non $A$ non $B$ hepatitis is still a concern.

With the licensure and availability of this new vaccine, the need for careful epidemiologic investigation and wellorganized hospital employee health services is increasing. Well-controlled studies to define the level of increased risk of HBV infection in hospital personnel are critically needed. Careful followup of large groups of vaccinated individuals for untoward effects over the short and long term are also necessary. Only after such data are collected can better, and more defensible, recommendations for the use of $\mathrm{HBV}$ vaccine in hospital personnel be made.

\section{REFERENCES}

1. Krugman S: The newly licensed hepatitis $B$ vaccine - Characterislics and indications for use. $J A M A 1982 ; 247: 2012-2015$.

2. Dienstag JL: Toward the control of hepatitis B. NEnglJ Med 1980; 303:874-76.

3. Hirschowitz BA, Dasher CA, Whitt FJ, et al: Hepatitis B antigen and antibody and tests of liver function - A prospective study of $\$ 10$ hospital laboratory workers. $A m J$ Clin Pathol 1980; 73:63-68.

4. Craig CP, Gribble C. Suarez K: Risk of hepatitis B among phlebotomists. Am J Infect Control 1981; 9:11-14.

5. Hepatitis Surveillance Report No. 47. Atlanta, Centers for Disease Control, December, 1981, p 3.
6. Purcell RH, Gerin JL: Hepatitis B vaccines. On the threshold. Am J Clin Pathol 1978; 70:159-169.

7. Kapikian AZ, Mitchell RH, Chanock RM, et al: An epidemiologic study of altered clinical reactivity to respiratory syncytial (RS) virus infection in children previously vaccinated with an inactivated RS vaccine. Am J Epidemiol 1969; 89:405-421.

8. Smith CB, Friedwald WT, Chanock RM: Inactivated $M$. pneumonia vaccine. Evaluation in volunteers. JAMA 1979; 199:853-358.

9. Fulginiti VA, Eller JJ, Downie AW, et al. Altered reactivity to measles virus: A typical measles in children previously immunized with inactivated measles virus vaccines. JAMA 1967; 202:1075-1080.

10. Szmuness W, Stevens CE, Harley EJ, et al: Hepatitis B vaccine. Demonstration of efficacy in a controlled clinical trial in a high-risk population in the United States. N Engl J Med 1980; 303:833-841.

11. Pattison CP, Maynard E, Berquist KR, et al: Epidemiology of hepatitis B in hospital personnel. Am J Epidemiol 1975; 101:59-64.

Bruce H. Hamory, M.D. Assistant Professor Department of Medicine Hospital Epidemiologist University of Missouri Health Science Center Columbia, Missouri

\section{Hepatitis B Vaccine: Its Risks and Benefits}

The advent of a vaccine to prevent hepatitis $B$ is the culmination of a 25-year explosion of knowledge concerning the disease. From the discovery of the Australia antigen to the development of a vaccine from an as yet uncultivable virus, this is the story of a triumph of medical research that equals other major breakthroughs in medicine this century.

One group to benefit will be medical workers, especially those who are exposed to blood and blood products. The vaccine comes at a time when we recognize that the problem of unidentified hepatitis carriers has increased sharply with the arrival in the U.S. over the past few years of immigrants from areas of the world where the disease is endemic. In addition, new medical technologies which rely increasingly on intravascular monitoring, expose more health care workers to more patients' blood than ever before.

Hepatitis $B$ is viewed by the average physician or nurse with the same fear that once was reserved for streptococcal infection in the pre-penicillin era. Everyone knows or has heard of someone who has died from the disease. So it is to an unusually receptive audience that the vaccine is offered. Past experiences with immunization programs for medical personnel have shown that they are apathetic if not overtly hostile toward plans to vaccinate them against rubella or influenza. ${ }^{1,2}$ Because of their fear of hepatitis, they are unlikely to react similarly in this case. Some of the concern about immunization has been founded in fact, but much has been misunderstanding of the risks involved. Therefore, it is equally important for us to examine the true risks and benefits of this vaccine from a scientific rather than an emotional standpoint.

The efficacy of the vaccine in a high-risk population is in little doubt. Its ability to prevent disease and sero-

Address reprint requests to: Peter N.R. Heseltine, M.D., Hospital Epidemiologist, Assistant Professor of Medicine, Los Angeles County University of Southern California Medical Center, 1200 North State Street, Box 596, Los Angeles, CA 90033. 